\title{
A Study on China's Financial Innovation and Industrial Structure Synergy Effects
}

\author{
Pengfei Zhu \\ School of Economics and Business, Beijing Jiaotong University, Beijing, China \\ flappypig@126.com
}

\begin{abstract}
Keywords: financial innovation; industrial restructuring; coordinative mechanism; VAR; degree of synergy.

Abstract: Have a collaborative mechanism analysis between financial innovation and industrial restructuring, and select time series data from 1993 to 2015 to build financial innovation, industrial structure adjustment comprehensive evaluation system and calculate by using principal component analysis, then build the VAR model, and carry out impulse response and variance decomposition; carry out empirical test about the synergies between financial innovation and upgrading of an industrial structure, based on coordination model to describe the collaboration characteristics between the two.
\end{abstract}

\section{Introduction}

Ding Chunling (2014) based on financial services to support the transformation and upgrading of industrial structure, carried out a research on the financial development and industrial restructuring research in Langfang City, to mention that the financial innovation was divided into financial system reform, financial markets, financial products, financial system and several other aspects of the innovation, etc., from these aspects to promote industrial restructuring in Langfang City [1]. Li Yuanyuan (2015) studied the mechanisms and principles of financial innovation in the industrial structure optimization and upgrades, they thought that financial innovation can act on industrial restructuring by direct path and indirect path in two ways [2].

\section{The Establishment of China's Financial Innovation and Industrial Structuring Adjustment Evaluation System}

2.1 Financial Innovation and Index System. In a comprehensive understanding of the connotation and characteristics of financial innovation and based on the existing research, measure the level of financial innovation in China from the stock market value/GDP 、 stock turnover/GDP 、 loans to financial institutions /GDP、 (M2-M1)/GDP、 (M2-M1)/GDP 、 total financial assets /GDP 、 central bank assets / total financial assets 、 Size of bank credit /GDP 、 total financial assets / trading financial assets。

2.2 Industrial Structure Adjustment and Target System. Industrial restructuring may use the proportion of non-agricultural industries, industrial structure factors, the ratio value between the tertiary industry and secondary industry, and Moore structure index, the proportion of high-tech industries and other indicators for measurement $[3,4]$.

\section{China's Financial Innovation and Industrial Structure Adjustment Collaborative Empirical Study}

3.1 Coordinative Mechanism. Whether it studies from the theoretical perspective, or the fact of the country's economic development, the adjustment of industrial structure and financial innovation have a synergistic effect [5]. On the one hand, financial innovation and industrial restructuring are interaction and mutual penetration and co-evolution. Each phase, each aspect of industrial restructuring needs the launch, guidance and maintenance of financial resources , the science and technology activities in the industrial restructuring are often accompanied by uncertainty, high risk and complexity, these features will largely affect the investment behavior of investors and the 
general direction of investment, therefore, it needs the suited financial service innovation to overcome the financial services needs brought by industrial restructuring; on the other hand, when the financial innovation transference and diffusion occur, traditional industrial sectors will be affected and needs upgrading, so the new industrial sectors along will be expanded, however, only change and innovation in financial services meet the needs of new industrial sector development. Thus, financial innovation and industrial restructuring are by co-evolution mechanism to realize economic growth [6]. The synergistic effect mechanism is: the synergistic mechanisms of the two are realized through two interrelated aspects: the first is that how industrial restructuring affects financial innovation, the second is how the financial innovation supports the changes of financial structure. Both focus on achieving an optimal degree of innovation and industrial structure, and this degree is the important condition that the two collaborate exercises synergistic effects.

\subsection{Interaction Relationship Research- based on VAR Model}

3.2.1 The OLS estimation results. The purpose of this study is based on the interaction between financial innovation and industrial structure adjustment, to explore the synergy degree between. Firstly, VAR model is used, particularly in the form of Eq. 1:

$$
y_{i}=\sum_{i=1}^{k} y_{i-1}+\mu_{i}
$$

Wherein, $\mathrm{T}$ is based on $2 \times 1$ vector of variables for time series data, $\mathrm{f}$ (comprehensive level of financial innovation) and i (industrial restructuring level) represent two endogenous variables in VAR model, t represents time, select the data from 1993 to 2015. VAR model OLS estimation results are shown in Table 3 and Table 1 below:

Table1 VAR model OLS estimation results

\begin{tabular}{lllllll}
\hline & $\mathrm{F}(-1)$ & $\mathrm{I}(-1)$ & $\mathrm{F}(-2)$ & $\mathrm{I}(-2)$ & $\mathrm{F}(-3)$ & $\mathrm{I}(-3)$ \\
\hline $\mathrm{F}$ & $-0.8682^{* * *}$ & $0.5809^{* *}$ & $-0.43804^{*}$ & $0.3981^{*}$ & $0.315^{*} 2$ & $-0.9721^{* * *}$ \\
$\mathrm{I}$ & -0.1687 & 0.1823 & $0.2167 *$ & 0.1873 & $0.2941^{*}$ & $-0.5342^{* *}$ \\
\hline
\end{tabular}

The estimation results show: financial innovation, industrial adjustment restructuring are mutual Granger causality under 5\% of significance. Financial innovations have significant influence on its own, industrial restructuring adjustment and the delayed impact of each stage, industrial restructuring adjustments also have influence on financial innovation.

3.2.2 The Short-term Interaction - the Impulse Response Function Analysis. In order to analyze the short-term dynamic effects between financial innovation and the upgrading of industrial structure, the impulse response function analysis is carried out, the results are shown in Fig. 1:
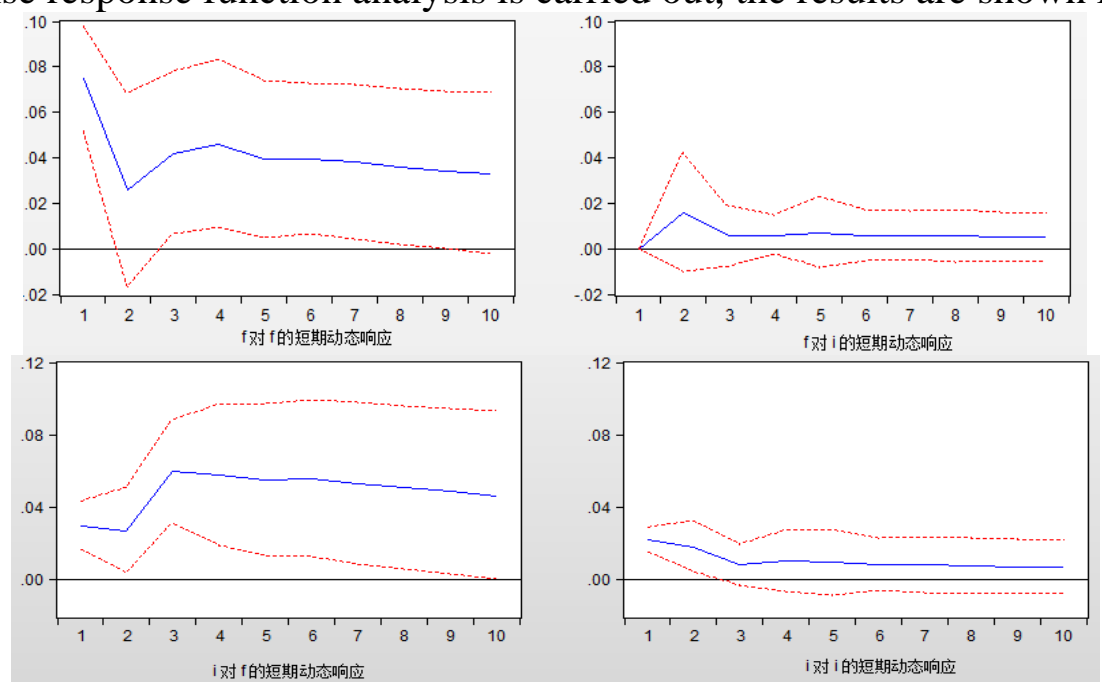

Fig.1 Impulse Response

The results can be seen from the figure: 1 ) after a standard deviation of impact to the current period of financial innovation, and the impact on their own future 10 are positive, indicating that the current level of financial innovation have co-relating effect of the latter part, and impact is 
maximum near the shock period, and then decreases; 2) the impact of financial innovation has a positive effect on the industrial structure adjustment, and the industrial structure level $\mathrm{i}$ is first to reduce then increase, and gradually stabilized, indicating that the impact level of financial innovation $\mathrm{f}$ to industrial restructuring i can promote the development of industrial restructuring in the short term level; 3) the impact of industrial restructuring has a positive effect on financial innovation, and it reaches a plateau over time; 4) just like the financial innovation, the impact of industrial restructuring will produce a positive impact on their own, there is a change in relation to the current period with the upgrading of industrial structure is expected to lag.

3.2.3 Long-term Interaction - Variance Decomposition. After a short term mechanism of action to financial innovation and industrial structure adjustment, to have a deeper and more complete understanding of the interaction between the two long-term VAR models, use variance decomposition to analyze the results, and the results are shown in Table 2:

Table 2 Variance decomposition results

\begin{tabular}{cccc}
\hline & $\mathrm{s}$ & $\mathrm{f}$ & $\mathrm{i}$ \\
\hline $\mathrm{f}$ & 10 & 0.9883 & 0.4751 \\
$\mathrm{i}$ & 10 & 0.2562 & 0.5933 \\
$\mathrm{f}$ & 20 & 0.8907 & 0.4623 \\
$\mathrm{i}$ & 20 & 0.3338 & 0.4747 \\
$\mathrm{f}$ & 30 & 0.7276 & 0.5342 \\
$\mathrm{i}$ & 30 & 0.4562 & 0.4148
\end{tabular}

According to the results of the variance decomposition, medium and long term the level of financial innovation, industrial restructuring impulse response levels were more than $70 \%$ and $50 \%$ of its own early. This shows that financial innovation and industrial restructuring on its own have a significant positive feedback phenomenon. From financial innovation and industrial restructuring to promote mutual relations, financial innovation for industrial restructuring industrial restructuring effect is greater than the role of financial innovation, but between the two mutual roles in promoting a significant presence.

3.3 Collaborative Degree between Financial Innovation and Industrial Restructuring. Record the overall score of financial innovation and the composite scores of industrial restructuring as $\mathrm{F}$ and I, refer to the synergies models and divide criteria [7], taking $\mathrm{K}$ equals to 3, using MATLAB to calculate the synergy degree between China's financial innovation and industrial restructuring from 1993 to 2015, and the results are shown in table3, wherein the degree of synergy calculation model is as Eq.2:

$$
\epsilon_{t}=\left|\frac{S_{1 t} \times S_{2 t}}{\left[\left(S_{1 t}+S_{2 t}\right) / 2\right]}\right|
$$

Table 3 Financial innovation and industrial structure adjustment of China’s synergy degree from 1993 to 2015

\begin{tabular}{cccc}
\hline Year & Synergy degree & Year & Synergy degree \\
\hline 1993 & 0.0030 & 2005 & 0.1252 \\
1994 & 0.0056 & 2006 & 0.1604 \\
1995 & 0.0062 & 2007 & 0.3238 \\
1996 & 0.0084 & 2008 & 0.1549 \\
1997 & 0.0125 & 2009 & 0.3875 \\
1998 & 0.0181 & 2010 & 0.3839 \\
1999 & 0.0650 & 2011 & 0.3045 \\
2000 & 0.0822 & 2012 & 0.3672 \\
2001 & 0.0877 & 2013 & 0.4288 \\
2002 & 0.1054 & 2014 & 0.5321 \\
2003 & 0.1518 & 2015 & 0.6071 \\
2004 & 0.1319 & & \\
\hline
\end{tabular}


Seen from the calculation results as a whole, from 1993 to 2015, the synergy degree between financial innovation and upgrading of industrial structure is not high, before 2006, financial innovation and industrial restructuring are basically incongruity; they barely reach the status of coordination between the two until 2013, and it has increased gradually in the past two years to gradually reach 0.5 or more.

\section{Conclusion}

The paper concludes from the following main aspects: 1) From the time dimension point of view, China's overall level of financial innovation and industrial restructuring increases year by year, the two has a similar development situation; 2) financial innovation and industrial structure adjustment are mutually in causal relationship, financial innovation has a certain role in promoting the development of industrial restructuring in the short term, and the promotion will be improved and stabilized in the long term; and industrial restructuring adjustment will promote the development of financial innovation in return in the medium and long term; 3) from 1993 to 2015, although the synergy degree between financial innovation and industrial restructuring shows an upward trend year by year, it is not high as a whole. Thus, in the process of realizing comprehensive, coordinative and sustainable economic development, we should deal with the relationship between financial innovation and industrial restructuring adjustment, financial services innovation should keep up with the pace of industrial restructuring to achieve mutual matching between the two.

\section{References}

[1] Ding Chunling, Liu Jing Financial Innovation in Promoting Industrial Restructuring and Upgrading Research [J] Shanxi University of Finance and Economics, 2014, (4): 31-32.

[2] Li Yuanyuan, Financial Innovation and Industrial Restructuring: Theory and Demonstration [J] Economic Issues, 2015, (3): 140-147.

[3] Ma Ning, Yan Taihua, Ji Xinlong Synergy Conditions, Analysis and Effects Test of Venture Capital and Intellectual Capital [J] Chinese J Management Science, 2015,3 (23): 24-31.

[4] Zhou Minglei, Ren Rongming Industrial Structure Sophistication and Energy Constraints [J] China Science and Technology Forum, 2011 (2): 105-111.

[5] Xie Xuemei Operation Mechanism of Synergistic Innovation: From a Metropolitan Perspective [J] Science Research, 2013, (12): 1907-1920.

[6] Ma Zhili Empirical Study of Financial Development to Industrial Structure Optimization [J] Finance and Economic Issues. 2011, (6): 10-14.

[7] Peng Yongzhang, Tao Zhangqi Empirical Study of Coordinated Development of Urban Construction and Upgrading of Industrial Structure [J] Jiangxi Normal University Journal, 2015, 3 (39): 263-269. 